\title{
SOLVING VENN-DIAGRAM BASED COMPREHENSION QUESTIONS IN SET THEORY USING NATURAL LANGUAGE PROCESSING
}

\author{
B Dinesh Reddy ${ }^{1}$, A Ravi Kiran ${ }^{2}$, Abhilash $^{3}$, N. Thirupathi Rao ${ }^{1}$ \\ and Debnath Bhattacharyya ${ }^{1}$ \\ ${ }^{1}$ Department of Computer Science and Engineering, \\ Vignan's Institute of Information Technology, \\ Visakhapatnam-530049, India \\ ${ }^{2}$ Sr. Member Technical, CDK Global India Pvt. Ltd. \\ ${ }^{3}$ Software Engineer, Envestnet Yodlee India \\ dinesh4net@gmail.com,1.akellaravikiran@gmail.com, \\ pradyumna.abhilash@gmail.com, nakkathiru@gmail.com, debnathb@gmail.com
}

Abstract-- Natural language problems have gained increased attention in the recent times. There are many special purposes, and carefully constructed evaluations driving the NLP research. Problem solving tests offer an interesting alternative to these evaluations. The primary goal of creating these problem solving tests is to evaluate the reading skills, and there by create bank of training materials and ranking procedures to match the existing measures of human performance. Solving Set Theory problems using NLP exposes once such research problem and helps creating an evaluation method for Natural Language Understanding Systems. This paper describes the possibility to challenge these systems to successively push higher performance levels up to an accuracy of $80 \%$ with high speed as an added advantage.

Keywords-- NLP, computer, human, set theory, skills, ranking, language, performance, accuracy

\section{INTRODUCTION}

The interaction between computer and humans was done in a machine understandable mode until Natural Language Processing (NLP) has started and gained precedence. Most of the modern applications still require the users to key in the system/language specific commands [1]. There are systems developed using interactive natural language commands that eases the user's interaction to provide commands in the natural language. Inducing the Natural Language understandability, processing and problem solving capacity to these systems makes all these systems AI-Complete [2, 3].

The maximum amount of data that is available on computer networks is unstructured data that can also be coined as Free Form text. The information from all this data should be extracted and processed in to be available for all the users [4]. NLP is one of the best approaches that can be applied to understand, extract and use the information from the unstructured data.

The main aim of Natural Language processing is solving problems like hypothesis testing, cross validation and baselines which include several techniques like lexicon

Received: February 11, 2019

Reviewed: April 8, 2019

Accepted: April 12, 2019

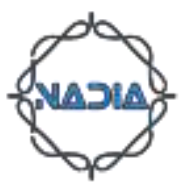


acquisition, word sense disambiguation, parsing, machine translation, and information retrieval [1]. This approach of processing brings great success to commercial sector. Some of the NLP systems aiming on Information Extraction, Question Answering and Bio-informatics use online encyclopedia [5]. Other systems attempt to understand the meaning of knowledge that is available in text form by deep study of word phrases, clauses and other elements of linguistics of the text to open up the concept of the text [6]. To achieve all these, strong integrated theories of cognitive science, human cognition and conceptual semantics are required.

There have been successful attempts to process natural language. The first attempt of Automation was Machine Translation (MT). One of the earliest projects on MT was started by Weaver and Booth in 1946 on computer translation based on expertise in breaking enemy codes during World War II $[7,8]$. The messages were in human language and were encrypted into various codes based on the time. The main aim of Machine translation at that time was to decrypt the encrypted messages. In 1957, Chomsky published Syntactic Structures that introduced the idea of generative grammar. After this, the field provided better insight into whether or how mainstream linguistics could help MT. During this period, other NLP application areas began to emerge. Right from processing simple text commands to interactive voice commands these systems have performed multiple tasks using NLP.

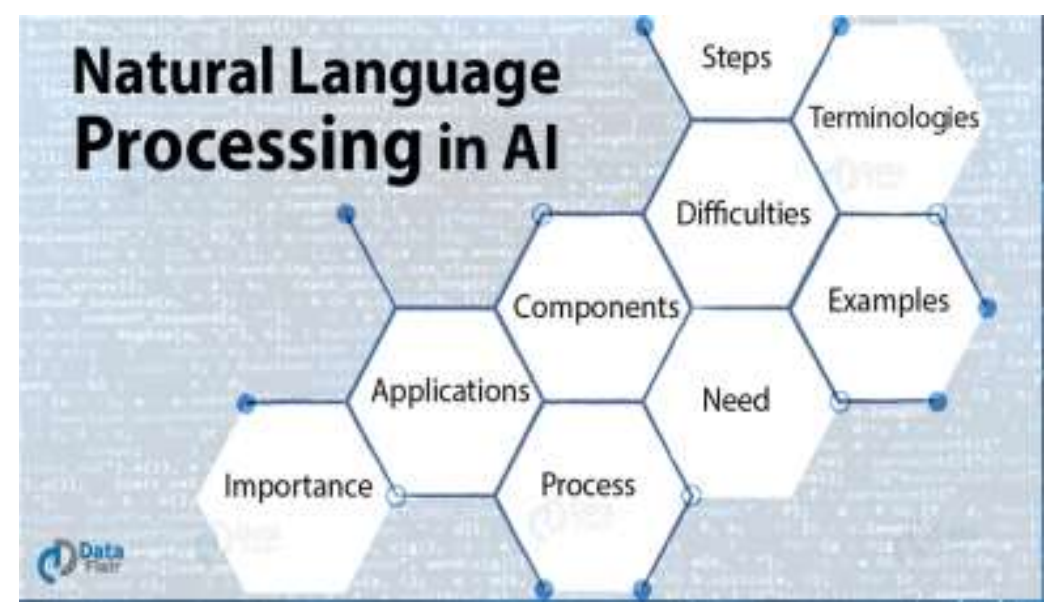

Fig.1 Natural Language Processing Model example [15]

Natural language processing is the new era of technology that always works and tries to improve the performance and always attempts to progression, understand and construct normal languages such as Hindi, English and Spanish etc. In real world situations, the natural language processing may not follow the actual rules or regulations developed or declared so far due to various factors. The major components of natural language processing systems are [15],

1. Natural Language Understanding

This method is used to convert and match the actual data was needed to be processed [15].

2. Natural language Generation

This method is carried out in three cases, they are planning of text, planning of sentences and realization of actual texts [15].

In general, this natural language processing applications and tools are worked in real time situations in combination with the artificial intelligence and its related techniques. Wherever the artificial intelligent techniques are being used such that to automate the 
process, the requirement of using AI and its related topics are used. Some of the common types of applications of NLP are as follows,

1. Optical Character Recognition: This application is mainly useful for the scanning purposes like banks, post offices and places where the actual addresses and data can be directly scanned and processed.

2. Speech Recognition: The recognition of speech is sometimes required in order to process several crime cases or sometimes it is useful for recognizing the voice for opening or closing of any sort of applications in mobile phones or some other security devices.

3. Machine Translation: In general, a person is required for translating a new language to the others when that particular person doesn't know about the language. But, by using this sort of applications, the translation of live speeches and other words of the languages are very easy to translate.

4. Sentiment Analysis: In some applications, the tastes or the likes and dislikes of the customers are needed to be calculated or to be analyzed. This process is mainly carried out only due to the increase of customers or increase of business for a particular company or any organization. Once the interests of the people or customers were analyzed, it is easy to attract the customers for further processing of such applications and brands for marketing.

5. Semantic Search: In order to find the similar topics or the similar items related to particular person or a particular place or other issues, this method of analysis is more important and can be used easily.

6. Machine Learning: These techniques and applications are used in combination with the NLP applications such that to analyze the applications more in detail and also to further process the data in a user friendly way such that the data can be easily processed with more efficient and better results.

Another research in this area was proposed in the paper "Answering Reading comprehension using Memory Networks" [9, 10, 11]. As per the approach proposed in the paper, memory networks play a very important role in answering a reading comprehension. The memory networks can be worked out in order to form neural networks which can efficiently draw a conclusion to a given problem $[8,9]$. In this approach, the problem of combining a memory component with an LSTM model for inference is solved by designing Supervised Memory Networks (or MemNNs) [10,12,13, 14]. This approach also included the training costs of the MemNN. This approach too was successful but the accuracy was still between 40-50\%.Going a step further, solving problems that are in Natural Language are not progressing at the same pace with commands.

The focus of this paper is around how the Venn-Diagram based Set Theory Problems can be solved using NLP.

\section{PROBLEM DESCRIPTION}

In this cognitive era every problem can be solved with the help of computers. The computers can think, evaluate and provide a solution to the problems that human beings have not thought of. If every problem could be solved using Computers, then why not mathematical problems? This question was the main motive of our problem description. So, what kind of mathematical problems we would consider in our work? Our work 
mainly discusses on how to evaluate the problems based on the Set Theory. Not all the problems in Set Theory can be solved using our work. But the mathematical problems which involve Venn diagrams could be solved using our work. Solving the Problems based on Venn Diagrams was not an easy task for a computer. The system had to read the input from the user, differentiate the data and the extract actual question from the input, and deduce the mathematical values from the questions to perform operations on them. Understand the question to come to a conclusion of what is the formula to be applied for the required solution. And then the conclusion is calculating and providing the output.

For example, if the problem statement is given as follows:

1) In a camp there are a total of 40 students. 32 students play only tennis. 5 students play only hockey.

*How many student play hockey?
A) 35
B) 32
C) 8
D) 40

Our work should be able to read the input from the user and understand the problem. In this case the total number of students the class $[\mathrm{N}(\mathrm{T} \cup \mathrm{H})]$ are 40 . Number of students playing only Tennis $[\mathrm{N}(\mathrm{T}-\mathrm{H})]$ are 32 . Number of students playing only Hockey $[\mathrm{N}(\mathrm{H}-\mathrm{T})]$ are 5. As we all know $\mathrm{N}(\mathrm{H} \cap \mathrm{T})=\mathrm{N}(\mathrm{H} \cup \mathrm{T})-(\mathrm{N}(\mathrm{T}-\mathrm{H})+\mathrm{N}(\mathrm{H}-\mathrm{T}))$. Our system should be able to deduce the mathematical formula, perform calculations and derive $\mathrm{N}(\mathrm{H} \cap \mathrm{T})$ as 3 . As per the question number of student who play hockey should be calculated suing the formula $\mathrm{N}(\mathrm{H})=\mathrm{N}(\mathrm{H}-\mathrm{T})+\mathrm{N}(\mathrm{H} \cap \mathrm{T})$ and output the answer to the problem as option $\mathrm{C}$

\section{METHODOLOGY}

There have been multiple QA systems in various areas. Search engines provide best question and answering system. A big focus in NLP has been to recognize natural language questions, extract the meaning, and provide the answer. With this system as motivation, we developed a system with the following proposed characteristics.

\section{PROPOSED SYSTEM}

The proposed system processed the input using Natural Language Processing (NLP) followed by tokenization, stemming and predicting the correct output based on cosine value. This proposed system provided correct predicted answer to the given question in the input with accuracy greater than $80 \%$. Below is the detailed description of each phases.

\section{ALGORITHMS AND TOOLS}

1. Natural Language Tool Kit: The NLTK is a toolkit developed by Stanford NLP. This is a platform that is processes human language data in order to construct Python programs to solve the specific problem. NLTK provides interfaces to resources that constitute more than 50 corpora and lexical resources, in addition to the suite of text processing libraries for classification, tokenization, stemming, tagging, parsing, and semantic reasoning [11]. The following functionalities of NLTK are adopted to be used in the proposed system are listed below.
a. Sentence segmenter
b. Word tokenizer
c. Parts-of-speech tagger [8] 
2. Porter Stemmer Algorithm: The morphological processing in NLP is performed in our proposed system using Porter Stemming Algorithm. This is a process to remove the suffixes from the English words. This removal eases the process of Information Retrieval. This algorithm stems the words and suffixes so that the actual input is converted to its root form for easier processing [2].

3. For example, if the description contains "Out of 40 students studying science 30 students are studying Chemistry and 20 students are studying Physics." Porter stemmer algorithm converts the word "Studying" to its root form i.e., "Study" to evaluate with the question, which might contain "How many students study only physics?" So that we obtain the accurate result while using the Cosine Similarity algorithm.

4. Cosine Similarity Algorithm: To measure the similarity between two entities, a metric is required. There are many coefficients that are identified and proposed like Jaccard coefficient, Cosine Similarity coefficient, Dice coefficient, Correlation, Hamming distance, Euclidean distance, and many more[7]. With studies saying Cosine Similarity Coefficient being suitable to the proposed architecture, and yielding better results [9][12][13], this is considered to obtain the relation between the tokens and sentences in human language form.

5. The Logic of Cosine Similarity is to convert the any given sentence into vectors and calculate the angle between them. Lesser the angle implies there exists relation between the vectors. If the Angle is 0 , then the vectors are strongly related. Hence "Lesser the Angle, Greater the Cosine Value, Greater the Similarity" [6].

\subsection{PROPOSED PROCESS ARCHITECTURE}

System architecture design for the proposed process abstracts the process to three main stages excluding the inputs text that contains the description and the question as a human language text and the output that is the solution to the question. The main aim of Machine translation at that time was to decrypt the encrypted messages. In 1957, Chomsky published Syntactic Structures that introduced the idea of generative grammar. After this, the field provided better insight into whether or how mainstream linguistics could help MT. During this period, other NLP application areas began to emerge. Right from processing simple text commands to interactive voice commands these systems have performed multiple tasks using NLP.

The data flows from input to the stage 1 for NLTK operations, then to stage 2 for stemming the root words using Porter's Stemmer and to the final stage where the data processed for to obtain the solution. The detailed architecture is described in the Figure 2 below. 


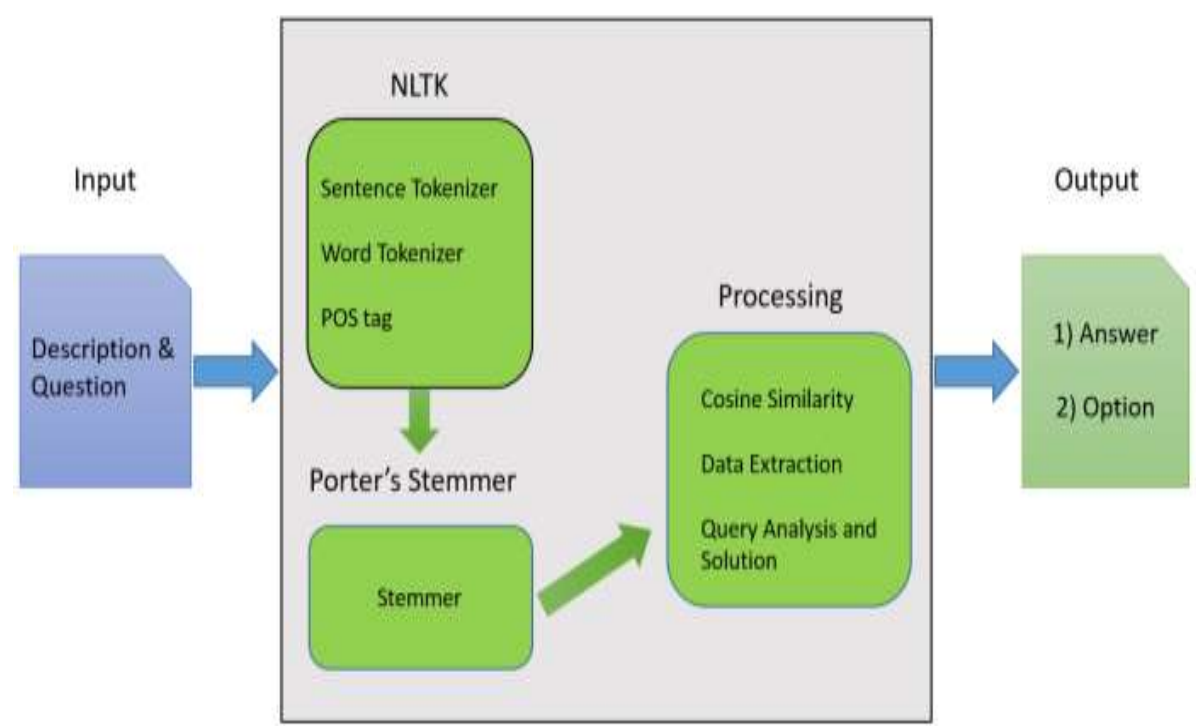

Fig. 2 Architecture of the Process Being Followed

The input data that is in Human Language is processed by using NLTK. In NLTK the input text is tokenized into sentences and then the sentences are tokenized to words. The words are further tokenized to stop words that are key words to be sent as input to stemming algorithm. The stop words are sent as input to the porter stemmer algorithm that stems the actual key word. The keyword dataset that contains the bag of desired words is compared with the tokens to identify the classed/Groups in the question using Cosine Coefficient. The numerical data is extracted and processed as follows in order to obtain the correct answer:

The data (raw data) obtained after extracting the root words by stemming, and categorizing under a particular part of speech is then processed to obtain the required data from it. Each sentence tokenized using the sentence tokenized is compared with questions provided using cosine similarity algorithm. Higher the similarity, greater is the probability of getting the desired output from the sentence.

This is compared with the options if provided and the correct option can be displayed. If the desired class doesn't exist in the data set, then it can be identified using the POS tag and added to the bag of words for further problem solving. The main aim of Machine translation at that time was to decrypt the encrypted messages. In 1957, Chomsky published Syntactic Structures that introduced the idea of generative grammar. After this, the field provided better insight into whether or how mainstream linguistics could help MT. During this period, other NLP application areas began to emerge. Right from processing simple text commands to interactive voice commands these systems have performed multiple tasks using NLP.

This process flow is described in detail in Figure 3. 


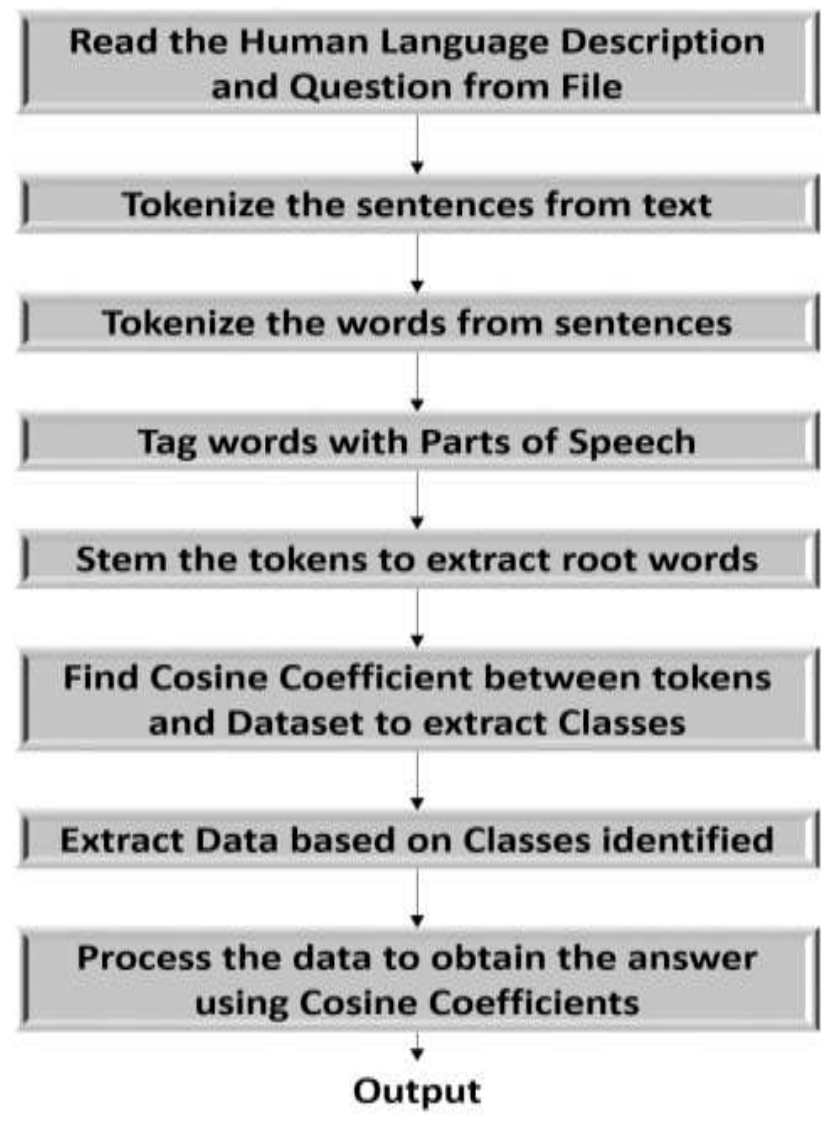

Fig. 3 Flow Chart of the System

Proposed system is designed to accurately predict answer to the given question in the input. This prediction is expected to give the correct answer with a probability of at least 0.8 . The entire process of the current system or the model can be observed clearly in the above figure 3 . In the first step, the data or the input from the users are collected by using the natural language processing techniques from the documents or the selected files. Then the data or the input data will be sent to the next step such that the data was processed by means of tokenizer. The sentences are tokenized from the entire text that was collected from $\mathrm{hr}$ document. Then the tokenized data was sent to the next step for further processing. The next step is to tokenize the words from the sentences and processed for further stages. Then the next phase of tagging the words tokenized with the selected parts of speech. In the next phase the tokens are made related with the root nodes or the root words of the documents or the languages. Then the Cosine coefficient was calculated between both tokens and datasets such that to extract the matched words or document contents. Then the set of classes in the datasets are identified and data is extracted. Then the final phase of the actual work was carried out that is the process of data to be obtained by using cosine coefficients. At last, the data required for further processing was calculated and can be analyzed for further utilization and for further matching with the actual data or the actual results to be required.

\section{EXPERIMENTAL RESULTS}

The evaluation of the current system is performed by applying the architecture and methodology on 70 Questions. The analysis and the checking of the current model performance were carried out in two phases or two steps. In first step, a question is given and associated questions related to that particular question was generated and the results are observed. Similarly, in the second step also the same process had been continued 
further. A question had been generated and based on that particular question, several other related questions are generated and the answers for the same questions had been generated and collected from the users. The output that was generated by the system and the outputs that were actual outputs both are studied and analyzed. The results are shown in the figure. It indicates that the system is able to predict the answer accurately for majority (74\%) of the questions. There always exist scenarios where the answers can't be derived due to insufficient data.

This approach also is not able to deduce them due to data insufficiency for the scenarios provided. This constitutes $18 \%$ of the scenarios considered. For the remaining $8 \%$ scenarios, this system could not predict the accurate answers. Upon careful analysis of these scenarios it is found that these cases failed due to the generalization. This system identifies the words as it is, but can't deduce the relation between them.

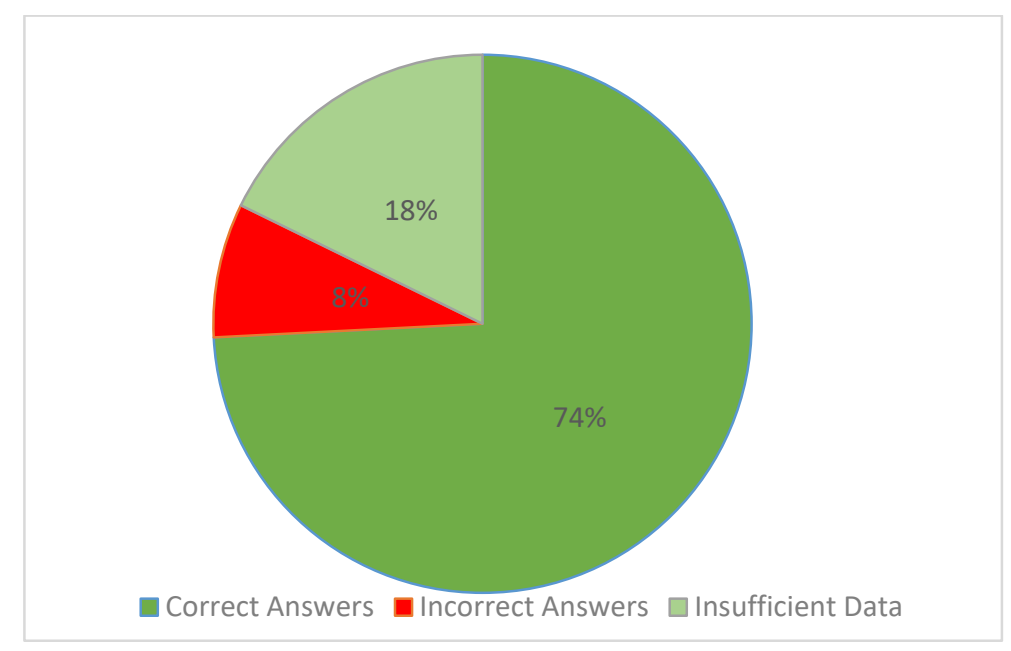

Fig. 4 Experimental Results

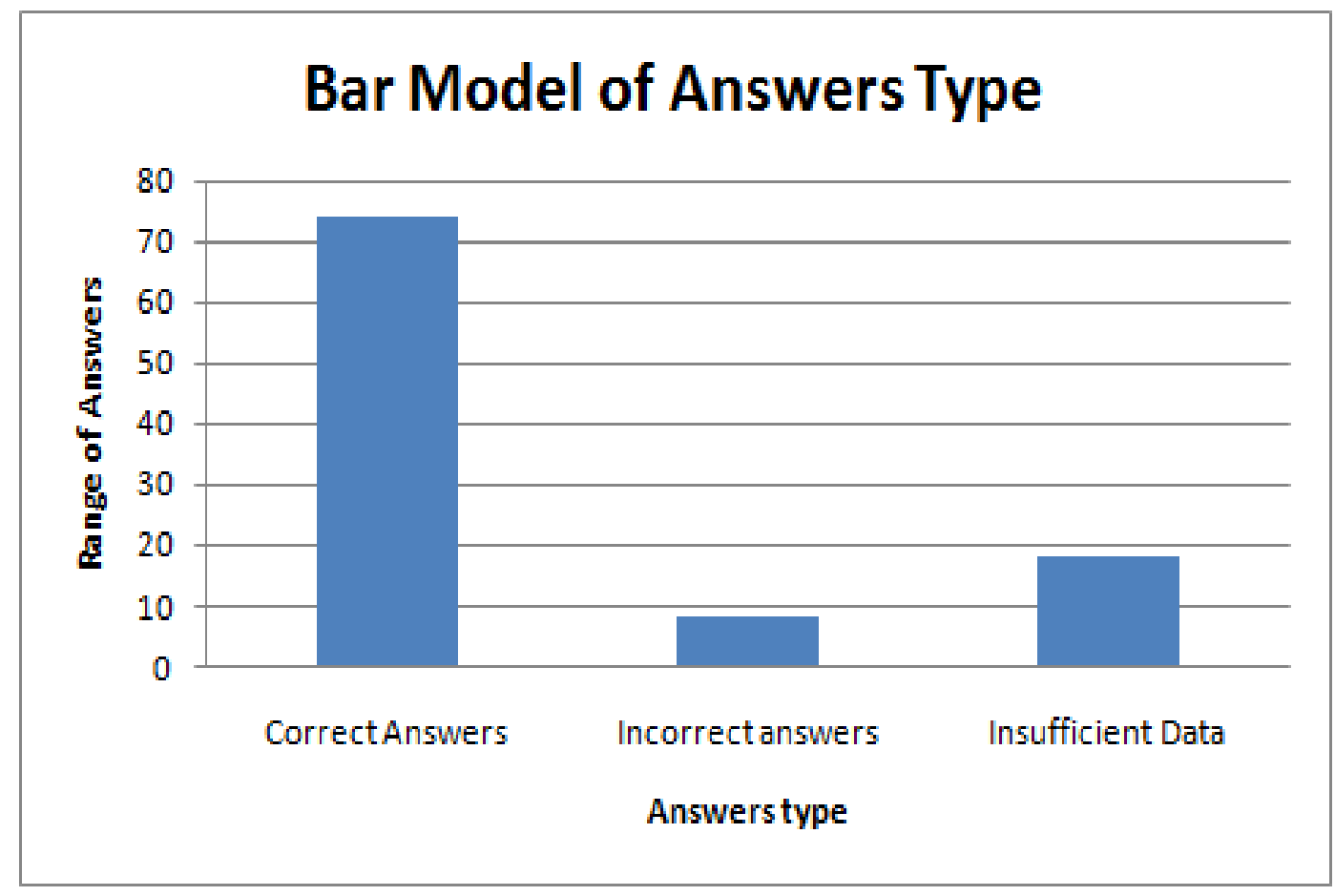

Fig. 5 Bar Graph model of Answers Types and Results 
The Sample questions with the expected and predicted answers are given below.

1. There exists a class of 30 students, the students talk in the language they study. Out of all the students in the class, 19 students study French. 12 students study Spanish and there are 7 students who study both French and Spanish.

Q1) What is the number of students who talk only Spanish?

Expected Answer: 5

Actual Answer: 5

Q2) How many students are talk neither French nor Spanish?

Expected Answer: 6

Actual Answer: 6

Q3) What is the number of students who talk only French?

Expected Answer: 12

Actual Answer: 12

Q4) How many students are do not learn any foreign languages?

Expected Answer: 6

Actual Answer: 0

2. Out of 50 elements in a set. $\mathrm{N}(\mathrm{A})=20 . \mathrm{N}(\mathrm{B})=15 . \mathrm{N}(\mathrm{A}$ and $\mathrm{B})=7 . \mathrm{N}(\mathrm{A}$ or $\mathrm{B})=28$

Q1) Find the number of elements in A or B?
A) 7
B) 13
C) 15
D) 8
E) None of the above

Expected Answer: None of the above

Actual Answer: None of the above

Q2) Find the number of elements in A?
A) 7
B) 13
C) 15
D) 8

Expected Answer: None of the above

Actual Answer: None of the above

Q3) Find the number of elements in B?
A) 7
B) 13
C) 15
D) 8
Expected Answer: C
Actual Answer: C

Q4) Find the number of elements only in A?
A) 7
B) 13
C) 15
D) 8
Expected Answer: B
Actual Answer: B

Q5) Find the number of elements in A and B?
A) 7
B) 13
C) 15
D) 8

Expected Answer: A

Actual Answer: None of the above

\section{CONCLUSION}

This work applied the techniques that are fast, accurate and require less cost. Unlike the existing methods like pattern matching which takes more time and Supervised MemNNs which require huge data sets for training, this approach is faster and highly 
efficient. The study and solution to the Venn diagram based problems that are in Human language is first of its kind. The earlier works on QA Systems on Natural Language were only successful for $60 \%$ of the time. The proposed system is proven to give effective results and less time to predict the expected output with accuracy of $75 \%$.

\section{FUTURE WORK}

Best functionality of the project is processing more number of text data and predicting the answer. This would however not be able to process the synonyms and generalized classes if any exists in the question. Ex: In a class of 25 members, there are 10 like apples, 20 like oranges. How many students like Fruits? We would try to enhance the project by processing all remaining set theory problems with accurate answers in future based on different calculative attributes.

\section{REFERENCES}

[1] Manning, Christopher D. and Hinrich Schütze. "Foundations of Statistical Natural Language Processing" Information Retrieval, 4, (2001), pp. 80-81.

[2] Kupiec, Julian, "MURAX: A robust linguistic approach for question answering using an on-line encyclopedia", In Proceedings of the 16th annual international ACM SIGIR conference on Research and development in information retrieval, ACM, (1993), pp. 181-190.

[3] Weaver, Warren, "Warren Weaver's memorandum in 1949: Translation. Milestones in Machine Translation", Locke, WN, Booth, AD (eds.) Ma-chine Translation of Languages: Fourteen Essays, (1955).

[4] Glenn Wadell et. al., "Two applications of Venn Diagrams", Teaching Statistics, Vol.33, No.2, (2011).

[5] Hirschman, L., Light, M., Breck, E. and Burger, J. D., "June. Deep read: A reading comprehension system", In Proceedings of the 37th annual meeting of the Association for Computational Linguistics on Computational Linguistics, (1999), pp. 325-332.

[6] Kapashi, Darshan and Pararth Shah, "Answering Reading Comprehension Using Memory Networks".

[7] Porter, Martin F., "An algorithm for suffix stripping”, Vol.14, No. 3, (1980), pp. 130-137.

[8] Huang, Anna, "Similarity measures for text document clustering", In Proceedings of the sixth new zealand computer science research student conference (NZCSRSC2008), Christchurch, New Zealand, (2008), pp. 49-56.

[9] The documentation and usage of NLTK is described in www.nltk.org. [Last Accessed on 20-02-2019].

[10] Thada, Vikas, and Vivek Jaglan, "Comparison of jaccard, dice, cosine similarity coefficient to find best fitness value for web retrieved documents using genetic algorithm”, International Journal of Innovations in Engineering and Technology, vol. 2, no. 4, (2013), pp. 202-205.

[11] Bird, Steven and Edward Loper, "NLTK: the natural language toolkit", In Proceedings of the ACL 2004 on Interactive poster and demonstration sessions, Association for Computational Linguistics, (2004), pp. 31.

[12] https://www.nltk.org/ [Last Accessed on 10-02-2019].

[13] https://stackoverflow.com/questions/945724/cosine-similarity-vs-hamming-distance. [Last Accessed on 10-02-2019].

[14] https://cmry.github.io/notes/euclidean-v-cosine. [Last Accessed on 10-02-2019].

[15] A blog entitled "What is Natural Language Processing in Artificial Intelligence?" by DATAFLAIR Team, November 2018. 